\title{
Formando grupos no internato: critérios de escolha, satisfação e sofrimento psíquico
}

\author{
Formation of groups during internship: \\ selection criteria, satisfaction, and psychological \\ distress
}

\author{
Mariana de Azevedo Gavioli ${ }^{\mathrm{I}}$ \\ Adriano Gonçalves Silva ${ }^{\mathrm{I}}$ \\ Rebeca Jesumary Gonçalves ${ }^{I}$ \\ Melina Erica Santos ${ }^{\mathrm{I}}$ \\ Keung Lin Shi ${ }^{\mathrm{I}}$ \\ Maria Cristina Pereira Lima ${ }^{\mathrm{I}}$
}

PALAVRAS-CHAVE

- Educação médica.

- Sofrimento psíquico.

- Estudantes de Medicina.

- Internato e Residência.

\section{KEY WORDS \\ - Education, Medical. \\ - Stress, Psychological. \\ - Students, Medical. \\ - Internship and Residency.}

\begin{abstract}
R E S U M O
Objetivo: Descrever os critérios para formação de grupos no internato e a avaliação que os alunos fazem do relacionamento grupal, analisando as variáveis associadas. Método: Trata-se de um estudo transversal, que utilizou questionário autopreenchido, com perguntas sobre dados sociodemográficos, autoavaliação do desempenho escolar, critérios para formação e satisfação com grupo de internato. Sofrimento psíquico foi avaliado a partir do Self Report Questionnaire. Utilizou-se o teste do qui-quadrado e regressão logística para análise multivariada. Resultados: A taxa de resposta no internato foi de mais de 90\%. A maioria dos alunos utilizou critérios ligados à rede social (82,6\%) e predominaram sujeitos satisfeitos com seu grupo (81,2\%). Após análise multivariada, apenas autoavaliação do desempenho escolar "boa ou ótima" e critérios de escolha relacionados à rede de apoio se mantiveram associados à satisfação com o grupo. Conclusões: Apesar de ser um estágio da formação profissional, os alunos se escolheram por questões ligadas à rede social. Sendo uma profissão na qual o trabalho em equipe é inerente, deveriam ser criadas estratégias durante o curso médico para elaborar as dificuldades de relacionamento grupal entre alunos de Medicina.
\end{abstract}

\section{A B S T R A C T}

Objective: To describe the criteria used in forming groups during medical internship and the students' evaluation of group relations, including analysis of the associated variables. Method: Cross-sectional study using self-completed questionnaires consisting of questions on socio-demographic data, self-rating of academic performance, selection criteria, and satisfaction with the group of interns. Psychological distress was assessed based on the Self-Report Questionnaire. The chi-square test and logistic regression were used for multivariate analysis. Results: Response rate among interns was over $90 \%$. The majority of the students used criteria related to the social network (82.6\%) and reported satisfaction with their groups (81.2\%). After multivariate analysis, only "good or excellent" self-evaluated academic performance and selection criteria related to the support network remained associated with group satisfaction. Conclusions: Although internship is a stage of professional training, in this study students chose each other based on reasons related to social networking. Given that teamwork is inherent to the medical profession, strategies should be created during the course to help students overcome their difficulties with group relations in order to reduce stress and improve training. 


\section{INTRODUÇÃO}

"O Inferno são os outros"

Sartre

O internato é considerado um momento especial do curso médico. Foi instituído em 1969 por resolução do Conselho Federal de Educação ${ }^{1}$ e desde o seu início já se caracterizava por propor treinamento essencialmente prático e intenso e ensino ministrado em pequenos grupos. O internato tem se modificado com a evolução da educação médica no país, que, por sua vez, tem procurado acompanhar as transformações na sociedade. Embora em algumas escolas o internato já contasse com estágios desenvolvidos nas comunidades há muitos anos ${ }^{2,3}$, na maior parte delas era quase exclusivamente hospitalar. Embora existam outras formas de internato, o mais comum é o rotativo, no qual os alunos realizam estágios em diferentes clínicas ou especialidades ${ }^{1}$.

A expectativa dos alunos em relação a esta fase do curso é grande. A respeito do início do internato, Leeder ${ }^{4}$ descreve que algumas escolas médicas manejam esta mudança com programas de orientação e tutoria para os alunos assim que eles chegam às enfermarias. Segundo Saadeh ${ }^{5}$.

Em termos profissionais é no quinto ano que o amadurecimento pessoal será posto à prova, pois, neste momento, define-se uma transformação no ensino, no aprendizado, na postura e nas vivências. (p.20)

Os alunos sabem que em seus estágios começarão a dar plantões e a assumir uma responsabilidade crescente com os pacientes. Provavelmente em função disto, a divisão dos alunos em pequenos grupos acaba sendo um processo intenso e, para muitos, estressan$\mathrm{te}^{6}$. Algumas faculdades formam seus grupos aleatoriamente ou ainda de acordo com uma relação alfabética. Na Faculdade de Medicina de Botucatu (FMB), assim como em outras escolas médicas ${ }^{6}$, os alunos fazem esta distribuição utilizando métodos e critérios escolhidos por eles. Frequentemente, esse processo é vivido com extremo sofrimento, em especial pelos alunos que se sentem preteridos pelos demais. No início do curso, os alunos mal se conhecem. No entanto, segundo Arruda ${ }^{7}$,

[...]à medida que os anos passam, vão se fazendo novas amizades, grupos anteriores dão lugar a novos agrupamentos, mas de maneira negativa vai ganhando corpo a marginalização de alguns estudantes, que passam a ser excluídos, vindo a formar aqueles agrupamentos denominados figurativamente por 'lixão (p. 65).

Sabe-se que diferentes manifestações de sofrimento psíquico têm se mostrado prevalentes entre estudantes de Medicina ${ }^{8-10}$, associando-se, muitas vezes, a fatores como rede de apoio ineficiente $^{11 .}$
Este estudo faz parte de uma ampla investigação sobre condições de vida e saúde de estudantes de Medicina, realizado na Faculdade de Medicina de Botucatu, sendo analisados aqui apenas os dados relacionados à formação de grupos e convivência nos grupos de internato. São objetivos deste trabalho: descrever os critérios para formação de grupos para o internato e a avaliação que os alunos fazem a respeito desse relacionamento grupal, analisando as variáveis associadas.

\section{MÉTODO}

Este estudo é parte de uma ampla pesquisa transversal, realizada na Faculdade de Medicina de Botucatu, na qual foram sujeitos todos os alunos matriculados do primeiro ao sexto ano, incluindo-se todos os presentes na sala de aula no momento da aplicação e que concordaram em participar. Foram excluídos do estudo apenas os estudantes que não se encontravam na sala no momento da aplicação do questionário. No presente estudo foram incluídos apenas alunos do internato (quinto e sexto anos).

\section{INSTRUMENTOS}

Foi utilizado um questionário anônimo, de autopreenchimento, aplicado em sala de aula aos estudantes que consentiram em participar. Foram investigadas informações sociodemográficas, bem como autoavaliação de desempenho escolar e saúde mental, entre outras. Foi também solicitado aos alunos que respondessem à seguinte questão aberta: "Quais critérios foram (ou serão) utilizados por você na formação dos grupos para o internato?".

Os alunos avaliaram também o relacionamento grupal em cinco categorias mutuamente exclusivas: muito bom, bom, regular, ruim e muito ruim.

Para avaliar sofrimento psíquico, foi utilizado o Self Reporting Questionnaire (SRQ), um instrumento desenvolvido pela $\mathrm{OMS}^{12} \mathrm{e}$ validado no Brasil por Mari e Williams ${ }^{13}$. Originalmente, o SRQ foi desenvolvido como instrumento de rastreamento para transtornos mentais comuns e possui 20 questões com respostas binárias, tipo "sim e não". As respostas às questões possibilitam estabelecer um escore, acima do qual o sujeito é considerado um provável caso. $\mathrm{O}$ ponto de corte utilizado foi diferente para homens e mulheres: seis ou mais respostas positivas para os homens e oito ou mais para as mulheres. Em função da alta sensibilidade (83\%) e especificidade (80\%) doSRQ, pesquisadores têm considerado os sujeitos que pontuam acima do ponto de corte como portadores de um "transtorno mental comum", uma condição que, embora não implique diagnóstico psiquiátrico formal, já indica um sofrimento psíquico relevante e potencialmente incapacitante. 


\section{Análise dos Dados}

As respostas às questões abertas foram digitadas e submetidas a pré-análise, que consistiu em leitura flutuante realizada por três autores (MAG, AGS e MCPL), de maneira independente. A partir da análise de conteúdo ${ }^{14}$, cada um estabeleceu uma categorização, depois apresentada aos demais. No final, as discordâncias foram discutidas, até se construir um consenso sobre as categorias finais, classificando as respostas quanto a estas. Foram obtidas três categorias: relativas à rede de apoio (que incluiu critérios como amizade, afinidade, convivência); relativas a desempenho como estudante (que incluiu competência, boas notas, responsabilidade); e uma categoria residual, outro, na qual foram agrupados critérios como: "exclusão de características negativas", "falta de opção", "prefiro não responder", "não escolhi", entre outros.

Foi elaborada uma planilha eletrônica, e os dados foram analisados por meio do Programa Stata 8.0. A variável dependente, "satisfação com o convívio grupal", resultou do somatório de "muito bom" e "bom" e do agrupamento das demais "regular", "ruim" e "muito ruim", sendo considerados, respectivamente, satisfatório e insatisfatório. As variáveis categóricas foram analisadas pelo teste do qui-quadrado ${ }^{15}$, sendo construído um modelo de regressão logística para as variáveis explanatórias que apresentaram p 0,15.

O projeto de avaliação das condições de vida e saúde mental, no qual este estudo se insere, foi aprovado pelo Comitê de Ética da Faculdade de Medicina de Botucatu em 8 de abril de 2002.

\section{RESULTADOS}

Foram devolvidos 455 questionários, o equivalente a uma taxa de resposta de $82,6 \%$, sendo que as características da população como um todo já foram descritas ${ }^{11}$. Para o momento, cabe destacar que houve predomínio de mulheres $(61,1 \%)$, de sujeitos com menos de 23 anos (75,2\%), de indivíduos que residem com colegas $(63,9 \%)$ e que gastam de três a quatro salários mínimos por mês.

Tabela 1

Satisfação com o convívio grupal entre alunos do internato da FMB-Unesp (n = 160)

\begin{tabular}{|c|c|c|c|c|c|}
\hline & \multicolumn{2}{|c|}{$\begin{array}{l}\text { Satisfatório } \\
129(81,2 \%) \\
\end{array}$} & \multicolumn{2}{|c|}{$\begin{array}{c}\text { Insatisfatório } \\
31(18,8 \%) \\
\end{array}$} & \multirow[t]{2}{*}{$\mathrm{p}^{*}$} \\
\hline & $\mathrm{n}$ & $\%$ & $\mathrm{n}$ & $\%$ & \\
\hline Rede social & 110 & 85,3 & 19 & 14,7 & \\
\hline Desempenho no papel de estudante & 7 & 46,7 & 8 & 53,3 & \\
\hline Autoavaliação do desempenho acadêmico & & & & & 0,01 \\
\hline Bom ou ótimo & 91 & 86,7 & 14 & 13,3 & \\
\hline Ruim ou muito ruim & 39 & 70,9 & 16 & 29,1 & \\
\hline Sim & 28 & 70,0 & 12 & 30,0 & \\
\hline Sentiu-se rejeitado & & & & & 0,04 \\
\hline Não & 97 & 85,8 & 16 & 14,2 & \\
\hline Sim & 33 & 71,7 & 13 & 28,3 & \\
\hline Sofrimento psíquico & & & & & 0,55 \\
\hline Ausente & 77 & 82,8 & 16 & 17,2 & \\
\hline
\end{tabular}

* Teste do qui-quadrado. 
A Tabela 1 apresenta as frequências de alunos satisfeitos e insatisfeitos com relacionamento grupal, segundo critério para formação de grupos e outras variáveis. Com relação ao convívio no grupo de internato, foram devolvidos 166 questionários, dos quais 6 $(3,6 \%)$ deixaram esta questão em branco. Entre os que responderam, $81,2 \%$ consideraram o relacionamento satisfatório, e 18,8\% insatisfatório. Os critérios relacionados à rede social apresentaram o maior percentual de sujeitos satisfeitos com o convívio grupal $(85,3 \%)$, seguidos da categoria "outros" (77,8\%). Entre os que men- cionaram critérios relacionados ao desempenho escolar, predominaram sujeitos insatisfeitos (53,3\%). Não houve associação com sexo $(p=0,81)$ e com sofrimento psíquico $(p=0,66)$. Foi observada associação com sentir-se rejeitado $(p=0,04)$, ter dificuldade para fazer amigos ( $p=0,03)$, com autoavaliação do desempenho escolar ruim ou muito ruim ( $p=0,01)$ e com critério para formação do grupo $(\mathrm{p}=0,001)$.

Tabela 2

Modelo de Regressão Logística para satisfação com convívio grupal entre internos da FMB-Unesp $(\mathrm{n}=160)^{*}$

\begin{tabular}{|c|c|c|c|c|c|}
\hline & OR bruta & IC $95 \%$ & OR ajustada & IC $95 \%$ & $\mathrm{p}$ \\
\hline \multicolumn{6}{|c|}{ Autoavaliação do desempenho acadêmico } \\
\hline Ruim ou muito ruim & 1,0 & & - & & 0,001 \\
\hline Bom ou ótimo & 2,6 & $1,2-5,9$ & 3,1 & $1,3-7,8$ & \\
\hline Critério para formação do grupo & & & & & 0,002 \\
\hline Desempenho como estudante & 1,0 & & - & & \\
\hline
\end{tabular}

*Incluídos apenas alunos do internato; sem informação de seis sujeitos.

Na análise multivariada, foram incluídos sentir-se rejeitado, ter dificuldade para fazer amigos, autoavaliação do desempenho escolar e critério para formação de grupo. Optou-se por excluir da análise multivariada o grupo "outros", por consistir em categoria bastante diversificada e com pequeno número de casos. Apenas critério para formação de grupo e autoavaliação do desempenho escolar permaneceram no modelo final, sendo que autoavaliação boa/ótima e critérios relativos à rede social associaram-se à satisfação, apresentando $\mathrm{OR}=3,1$ e $\mathrm{OR}=7,3$, respectivamente.

\section{DISCUSSÃO}

No presente estudo, observou-se que, apesar do internato representar uma fase de treinamento em serviço, os critérios de escolha relatados pelos alunos são predominantemente relacionados à rede social. Acredita-se que a convivência em grupos seja inerente ao ser humano. A partir de uma análise dos textos de
Sartre, Bettoni e Andrade ${ }^{16}$, afirmam que: "ao estar-no-mundo, o homem é automaticamente forçado, em sua práxis, a se relacionar com as coisas, com os outros, consigo mesmo e principalmente com as instituições" (p. 67). Isto é especialmente verdadeiro para os médicos, de quem se espera capacidade de comunicar-se adequadamente com seus pacientes e trabalhar em equi$\mathrm{pe}^{17}$. Segundo Moreno ${ }^{18}$, o homem não pode ser concebido sem que esteja em relação com o mundo e, em particular, com outro homem:

Nenhum individuo permanecerá isolado do outro, nem nenhum grupo permanecerá separado de outros se viverem geograficamente próximos. Mais cedo ou mais tarde realizarão intercâmbio de emoções e de outros valores sociais[...] (p. 92).

Bellodi et al. ${ }^{19}$, a partir da discussão do tema "formação de grupos (panelas) para o internato", nas reuniões do programa de 
Tutoria da FMUSP, observaram que os alunos preferem a livre escolha como critério para esta divisão. De maneira semelhante ao que ocorreu no presente estudo, os critérios "amizade" e "responsabilidade" foram citados como essenciais para a composição dos grupos. Os autores chamam a atenção para a importância das escolas médicas estarem atentas não só às necessidades de aprendizagem dos alunos, mas também às suas necessidades emocionais. Embora não se possa discordar de que o processo de formação de grupos e a convivência estreita nestes anos do internato sejam experiências com intensa carga emocional, apresentam grande potencial no que diz respeito à aprendizagem.

Em um estudo de validação de instrumento para avaliar trabalho em grupo, Sobral ${ }^{20}$ notou que alunos que desenvolveram atividades acadêmicas em pequenos grupos obtiveram pontuações melhores do que aqueles que não o fizeram. Tal escala considera aspectos como "preservação do grupo", "trabalho em equipe" e "disposição para ajudar", entre outros. O ensino em pequenos grupos, como o que ocorre no internato, pode ter um papel importante na formação médica, desde que as dificuldades surgidas na dinâmica grupal possam ser trabalhadas. As escolas médicas têm mostrado um interesse crescente em oferecer diferentes formas de suporte grupal para seus alunos ${ }^{5,21}$, mas há poucas experiências que abordaram a dinâmica intragrupal. Em um trabalho realizado na FMB, Fonseca et al. ${ }^{22}$ observaram que havia demanda para o trabalho de supervisão das relações intragrupo, no qual foi possível identificar as dificuldades e aumentar a coesão grupal.

Uma questão muito presente no internato e que merece atenção é a competitividade que se acirra especialmente ao final, pela proximidade com o exame de residência ${ }^{7}$. Como o questionário foi aplicado aos alunos em junho, é possível que os sextanistas ainda não estivessem no auge do estresse com o exame de residência. Em função disto, não só a convivência estaria mais tranquila, mas também o modo como avaliaram o relacionamento poderia ter sido mais condescendente.

Outro aspecto metodológico que precisa ser mencionado é o número de sujeitos incluídos neste estudo. Embora represente mais de $90 \%$ dos alunos matriculados no internato, limita as análises estatísticas, em especial a análise multivariada. Seria interessante que outras pesquisas, com número maior de alunos por turma ou associando diferentes escolas, procurasse analisar o relacionamento dos alunos não só no internato como em outros períodos do curso.

Não ter sido encontrada relação com TMC é bastante instigante e exige reflexão. É possível que o sofrimento de alguns alunos seja de fato relevante, mas individual. Estudos qualitativos seriam interessantes para observar de perto este processo de organização dos alunos nos pequenos grupos. Não só auxiliariam a identificar estratégias de coping, como também poderiam ex- plorar o significado dos critérios empregados pelos alunos. Esta é uma interessante questão de pesquisa que se coloca, porque a forma como os alunos se relacionam entre si e com seus professores pode ter consequências bastante adversas, caracterizando estressores evitáveis do curso médico ${ }^{23}$. Instrumentos como o teste sociométrico ${ }^{18}$ ou mesmo a supervisão de grupos poderiam ser utilizados nos processos de rearranjo e convivência de grupos de alunos ao longo do curso médico. Estas estratégias não só poderiam auxiliar na elaboração de conflitos intragrupais, como permitiriam identificar e trabalhar dificuldades de relacionamento específicas dos estudantes.

\section{CONSIDERAÇÕES FINAIS}

Embora estudos transversais não possibilitem falar em causalidade, a associação dos critérios relacionados à rede social com uma boa avaliação das relações grupais sugere que este critério não deveria simplesmente ser deixado de lado quando da divisão em grupos. Oferecer diferentes possibilidades de os alunos interagirem entre si ao longo do curso, lidando com as dificuldades encontradas, poderia aumentar a rede de relacionamentos, auxiliando inclusive sua saúde mental.

Na maior parte das escolas médicas, as turmas são grandes, e há pouca ou nenhuma oportunidade de ampliar os contatos com os próprios colegas. Supervisões voltadas para o relacionamento grupal poderiam auxiliar os estudantes no desenvolvimento de suas habilidades para o trabalho em equipe, preparando-os para o futuro profissional. É importante que não se caia no falso maniqueísmo de "livre escolha" versus "método aleatório", discutindo qual forma é a melhor para compor os grupos. Mais interessante seria aproveitar as experiências de formação e convivência grupal, próprias do internato, como um cenário privilegiado de aprendizagem, para desenvolver a aprimorar a habilidade do trabalho em equipe e, por que não dizer, de relacionamento humano.

\section{REFERÊNCIAS}

1. Marcondes E, Mascaretti LA. O internato na graduação médica. In: Marcondes E, Gonçalves E.L. (Org.) Educação Médica. São Paulo: Savier; 1998. p.149-66.

2. Magaldi C, Giarola LC, Oliveira LR. O ensino de saúde pública na graduação: o internato rural (1970-1985). In: Cyrino AP, Magaldi C (Org). Saúde na Comunidade: 30 anos de experiência em extensão universitária em saúde coletiva. Botucatu: Cultura acadêmica; 2002. p.150-173.

3. Polignano MV, Melo EM, Nascimento LE, Santana AMP, Rodrigues Jr IA. Internato Rural da Faculdade de medicina: 25 anos de integração docente-assistencial. [online] Anais do 7º Encontro de extensão da Universidade Federal de Minas gerais; 2004 Set. 
12-15 [acesso em: 22 dez. 2007]; Belo Horizonte. Disponível em http://www.ufmg.br/proex/arquivos/7Encontro/Educa107.

4. Leeder SR. Preparing interns for practice in the 21st century. MJA 2007;186:S6-8.

5. Saadeh A. Internato em medicina: estudo da interação estudante-paciente. São Paulo; 1995. Mestrado [Dissertação] - Universidade de São Paulo.

6. Marcondes E. Distribuição dos alunos pelos grupos de internato: uma questão importante. Boletim Ponto e Vírgula (CEDEM).1993;24:4-6.

7. Arruda PCV. As relações entre alunos, professores e pacientes. In: Milan LR, De Marco OLN, Rossi E, Arruda PCV. O Universo psicológico do futuro médico: vocação, vicissitudes e perspectivas. São Paulo: Casa do Psicólogo;1999. p.43-73

8. Porcu M, Fritzen CV, helber C. Sintomas depressivos nos estudantes de medicina da Universidade Federal de Maringá. Psiquiatr Prat Méd. 2001;34:2-6.

9. Souza FGM, Menezes Neto GC. Estresse nos estudantes de Medicina da Universidade Federal do Ceará. Rev Bras Educ Med. 2005;29:91-96.

10. Moro A, Valle JB, Lima LP. Sintomas depressivos nos estudantes de medicina da Universidade da Região de Joinville (SC). Rev Bras Educ Med. 2005;29:97-102.

11. Lima MCP, Domingues MS, Ramos-Cerqueira ATA. Prevalência e Fatores de risco para transtornos mentais comuns entre estudantes de medicina. Rev Saúde Publica. 2006;40:1035-1041.

12. Harding TW, Arango MV, Baltazar J, Climent CE, Ibrahim HHA, Ignácio LL, Murthy, RS, Wig NN. Mental disorders in primary health care: a study of their frequency and diagnosis in four developing countries. Psychol. Med. 1980;10:231-241.

13. Mari JJ, Willians PA. comparison of the validity of two psychiatric screening questionnaires (GHQ-12 and SRQ-20) in Brazil, using Relative Operating Characteristic (ROC) analysis. Psychol. Méd. 1985;5:651-659.

14. Bardin L. Análise de Conteúdo. Lisboa: Edição 70; 1977.

15. Hennekens $\mathrm{CH}$, Buring JE. Epidemiology in Medicine. Boston: Little, Brown, and Company; 1987.
16. Bertoni RA, Andrade MJN. Formação dos grupos sociais em Sartre. Revista Eletrônica. 2002;4:67-75.

17. Almeida M. Diretrizes Curriculares Nacionais para os cursos universitários da área da saúde.Londrina: Rede Unida; 2003.

18. Moreno JL. Psicoterapia de grupo e psicodrama. Introdução à teoria e à práxis. São Paulo: Mestre Jou; 1974.

19. Bellodi PL, Chebabo R, Abensur SI, Martins MA. Grupos no Internato: panela, caldeirão ou frigideira? Rev Bras Educ Méd [CD-ROM]. 2007;31(supl 1):77. [Anais do 45 Congresso Brasileiro de Educação Médica].

20. Sobral DT. Desenvolvimento e uso de medida do trabalho em grupo na aprendizagem cooperativa: medida do trabalho em grupo. Rev Bras Educ Méd. 1997;21:7-12.

21. De Marco OLN. Grupos de reflexão com quintanistas de medicina. In: Milan et al. O Universo Psicológico do Futuro Médico. São Paulo: Casa do Psicólogo; 1999. p.129-138.

22. Fonseca NMV, Megid JL, Ramos-Cerqueira ATA, Lima MCP. Grupo de Integração do Quinto Ano: uma tentativa de trabalho psicoprofilático junto às equipes de internato. Anais do 20 Congresso Brasileiro de Psiquiatria. p.417.

23. Costa LSM, Pereira CAA. O abuso como causa evitável de estresse entre estudantes de medicina. Rev Bras Educ Med. 2005;29:185-190.

\section{CONFLITO DE INTERESSES}

Declarou não haver.

\section{ENDEREÇO PARA CORRESPONDÊNCIA}

\section{Maria Cristina Pereira Lima}

Departamento de Neurologia e Psiquiatria

Faculdade de Medicina de Botucatu - Unesp

Rubião Junior, $\mathrm{s} / \mathrm{n}$

CEP.: 18618-000 - Botucatu - SP

Cx. P. 540

E-mail: mclima@fmb.unesp.br 